\title{
INFLUENCIA DEL ESTILO DE APRENDIZAJE EN EL RENDIMIENTO ACADÉMICO DE ESTUDIANTES UNIVERSITARIOS
}

\section{INFLUENCE OF THE LEARNING STYLE ON THE ACADEMIC PERFOR- MANCE OF UNIVERSITY STUDENTS}

\author{
TANIA BRIONES LINARES \\ Universidad Privada Norbert Wiener \\ Judith Soledad Yangali Vicente \\ Universidad Privada Norbert Wiener
}

\section{RESUMEN}

Una de las estrategias educativas que cada docente debería poseer es saber identificar los estilos de aprendizaje de cada estudiante, ya que estos pueden influir directamente en lograr un buen rendimiento académico. El objetivo de la investigación fue determinar la relación entre los estilos de aprendizaje y los niveles del rendimiento académico en los cursos del área de Microbiología de estudiantes de la especialidad de Laboratorio Clínico y Anatomía Patológica de la Universidad Alas Peruanas del semestre académico 2019-1. La investigación fue de tipo básica con enfoque cuantitativo de alcance correlacional y diseño no experimental. La muestra constó de 102 estudiantes, los datos fueron procesados en SPSS 26.0 y la contrastación de hipótesis con el Coeficiente de Cramer. A cada estudiante se le aplicó el Inventario de Estilos de Aprendizaje de Kolb, encontrándose que el 36,3\% tienen preferencia por el estilo asimilador, el 26,5\% el estilo acomodador, el 20,6\% el estilo convergente y $16,7 \%$ el estilo divergente. Con respecto a los estilos de aprendizaje y los niveles del rendimiento académico, se obtuvo el estadístico de prueba $\mathrm{V}=$ 0,236 y la significación aproximada de 0,049 , por lo que se concluye que el estilo de aprendizaje tiene una relación débil con el nivel de rendimiento académico de los estudiantes.

Palabras claves: Convergente, divergente, acomodador, asimilador.

97 
Influencia del estilo de aprendizaje en el rendimiento académico de es-

Abstract

One of the educational strategies that each professor must possess is knowing how to identify the learning styles of each student since these can directly influence in achieving good academic performance. The objective of the research was to determine the relationship between learning styles and levels of academic performance in courses of the Microbiology Area in Clinical Laboratory and Pathological Anatomy's students of the Alas Peruanas University from the academic semester 20191 . The research was a basic type with a quantitative approach of correlational scope and non-experimental design. The sample consisted of 102 students, the data was processed in SPSS 26.0 and the hypothesis was tested with the Cramer's Coefficient. The Kolb Learning Styles Inventory was applied to each student, finding that $36,3 \%$ prefer the assimilator style, $26,5 \%$ the accommodator style, $20,6 \%$ the convergent style and $16,7 \%$ the divergent style. Regarding the learning styles and the levels of academic performance, the test statistic $\mathrm{V}=0,236$ and the approximate significance of 0,049 were obtained, and it was concluded that the learning style has a weak relationship with the level of academic performance of the students.
Key words: Convergent, divergent, accommodating, assimilator.

\section{INTRODUCCIón}

La educación es la piedra angular de todas las naciones, ya que su desarrollo es medible por el nivel de educación de los ciudadanos, según Keefe y Ferrell (1990), citados por Chanataxi (2019), los problemas de aprendizaje con frecuencia no están relacionados con la dificultad del tema sino con el tipo de aprendizaje. El estilo de aprendizaje por sí solo no es el único factor que puede influir en una situación de aprendizaje, se debe tener en cuenta el contexto educativo y cultural de la universidad, la conciencia individual, la experiencia de vida, otras habilidades de aprendizaje, efecto del educador, motivación, etc. (Sarmiento 2007). Pashler, et al (2008) citado por İlçin, et al. (2018) demostraron que prácticamente no había evidencia de que las personas aprenden mejor cuando el estilo de enseñanza se adapta al estilo de aprendizaje preferido de los estudiantes.

Las ideas constructivistas del conocimiento indican que el aprendizaje se logra a partir de la experiencia; es decir, los seres vivos deben crear significados por su propia experiencia, esta 
adquisición del conocimiento es activa (Tünnermann, 2011). La teoría explica cómo cada individuo, estudiante para el caso, adquiere conocimiento y busca aplicarlos en la solución de problemas. Ello les permite construir su comprensión de una determinada situación y pasará a formar parte de su experiencia en relación entre conocimiento, aprendizaje y enseñanza es una consecuencia de la interdependencia entre procesos individuales y sociales en la construcción de este; de acuerdo con Vygotsky (1986) esto se logra interrelacionando la mente, el lenguaje y el pensamiento, el habla externa e interna, la naturaleza y la cultura, así como los procesos sociales e individuales.

Es valioso el aporte de Cabrera y Fariñas (2019), quienes afirman que el estudio de los estilos de aprendizaje se está entendiendo como un proceso de percepción y procesamiento de la información; esto da una importancia enorme al conocimiento, a lo intelectual, a la acumulación de información sobre lo afectivo-emocional, lo experimental. Kolb (1984), Kolb y Kolb (2013) explican que el modelo de estilos de aprendizaje de Kolb está respaldado por la teoría de aprendizaje en base a experiencias de Kolb (ELT), una teoría integral del aprendizaje y el desarrollo de adultos; explican que ELT se basa en las propuestas de algunos eruditos prominentes, a saber, John Dewey, Kurt Lewin, Jean Piaget, Lev Vygotsky, William James, Carl Jung, Paulo Freire, Carl Rogers y Mary Parker Follet; de acuerdo con estos, el aprendizaje debe considerarse un proceso y no solo por los resultados obtenidos. En el postulado histórico-cultural del aprendizaje en el que se inserta la teoría, Kolb (1984) utiliza el concepto vygotskiano de zona de desarrollo proximal para fundamentar un nuevo concepto, el «aprendizaje experimental», dirigido al aprendizaje de adultos. Kolb detecta los siguientes estilos:

- Divergente: el estilo de aprendizaje derivado de la combinación CE / RO. Las personas con este estilo tienen preferencia por los estímulos visuales, situaciones concretas, combinadas con información diversa. Se sienten cómodos con el trabajo grupal, la discusión y la retroalimentación constante.

Asimilador: se caracteriza por la preferencia por los estímulos visuales y mentales ( $\mathrm{RO} / \mathrm{AC}$ ). Los estudiantes con este estilo manejan más fácilmente el análisis, las explicaciones, las teorías, los textos y todo tipo de material que permita el análisis y la reflexión. 
- Convergente: es el estilo de aprendizaje de las personas que se identifican con tareas prácticas y razonamiento deductivo para resolver un problema determinado (AC / $\mathrm{AE})$. Estos alumnos prefieren la orientación directa y práctica y las tareas de aprendizaje.

- Acomodador: es el estilo de aprendizaje identificado por la preferencia de hacer planes, proyectar el futuro, crear perspectivas para situaciones, a partir de estímulos que implican pensar y hacer (AE / CE). Las personas con este estilo manejan actividades desafiantes fácilmente, toman riesgos y resuelven problemas de forma intuitiva.

El rendimiento académico sin duda se ve influenciado por diversos factores, genéticos, entornos familiares y de la comunidad, económicos y políticos, y sin duda cada uno de ellos tendrá una influencia en diferente grado sobre el trabajo académico de cada estudiante. Sin embargo, como lo dice Francis y Segun (2008), el ambiente académico y los factores relacionados con la docencia son los que más influyen en los logros académico, especialmente si cada estudiante tiene una elevada automotivación. En una economía globalizada, Revista de Investigación de la Universidad Norbert Wiener, 2020, N. 9 con situaciones como pandemias que pueden afectar directamente la capacidad de resiliencia familiar, se busca que cada estudiante ponga en práctica las competencias que logró en el centro de formación profesional, y cada uno de estos debe asegurar a la sociedad formar profesionales resilientes que actúen con soluciones prácticas en su comunidad, pero para ello es importante que cada docente sepa cómo aprende cada estudiante, explotar sus estilos de aprendizaje, ya que estos influirán en los logros académicos.

Esta investigación busca determinar la relación entre los estilos de aprendizaje y los niveles del rendimiento académico en los cursos del área de Microbiología de estudiantes de la especialidad de Laboratorio Clínico y Anatomía Patológica de la Universidad Alas Peruanas del semestre académico 2019-1. La importancia al investigar los estilos de aprendizaje fue identificar las necesidades de cada estudiante y proponer estrategias de aprendizaje enfocadas en los estilos de estos para lograr los objetivos propuestos en cada sesión de clase. El rendimiento académico, así como la autoconfianza de cada estudiante aumentarán al elaborarse cursos basados en sus estilos de aprendizaje. La hipótesis propuesta en esta investigación fue demostrar la relación significativa entre 100 
los estilos de aprendizaje y los niveles del rendimiento académico.

\section{MATERIAL Y MÉTODO}

Este trabajo de investigación es de tipo básico porque busca incrementar conocimientos en el proceso de la enseñanza-aprendizaje. El alcance es correlacional porque su objetivo es evaluar la relación que existe entre dos o más variables. El diseño es no experimental ya que no se manipuló deliberadamente variables, se observó los fenómenos tal y como se dan en su contexto natural, para después analizarlos; en este diseño no fue posible asignar aleatoriamente a los participantes o tratamientos porque no hay condiciones o estímulos a los cuales se expongan los sujetos del estudio. La población estuvo conformada por 138 estudiantes regulares matriculados en los cursos de Parasitología, Micología, Microbiología sanitaria y Diagnóstico laboratorial del área de Microbiología del quinto al octavo ciclo de Laboratorio Clínico y Anatomía Patológica de la Universidad Alas Peruanas del semestre 2019-1.

El número de la muestra se obtuvo aplicando la fórmula para una población finita (138):

Donde: 101
- $\mathrm{N}$ = Total de la población (138)

- $\mathrm{Za}=1.96$ al cuadrado (si la seguridad es del 95\%)

- $\mathrm{p}=$ proporción esperada (en este caso $50 \%=0.5)$

- $\mathrm{q}=1-\mathrm{p}$ (en este caso 1-0.5 = 0.5)

- $d=$ precisión (un 5\%)

De esto, se obtuvo una muestra conformada por 102 estudiantes.

Para determinar el estilo de aprendizaje se usó la técnica de la encuesta, y el instrumento Inventario de Estilos de Aprendizaje (IEA) de Kolb, el cual evalúa la preferencia por un estilo de aprendizaje: divergente, asimilador, convergente y acomodador. Este inventario consta de 12 oraciones que deben completarse con opciones en orden jerárquico; hay cuatro opciones distintas que son 1, 2, 3 y 4 , siendo este último el más característico y va disminuyendo hasta el 1 que no es nada característico. Una vez que el estudiante completó el inventario, se colocó la plantilla, la cual identifica los números con su correspondiente fase. Las fases son Experiencia Concreta (EC), Observación Reflexiva (OR), Conceptualización Abstracta (CA) y Experimentación Activa (EA); luego se suma los puntajes de cada una de las fases. Con los totales obtenidas se realizan las siguientes restas: $\mathrm{CA}$ - EC $=$ CA-EC y EA - OR = EA-OR. Am- 
Influencia del estilo de aprendizaje en el rendimiento académico de es-

tudiantes universitarios

bos resultados obtenidos de las restas se con Microsoft Excel 2019 e IBM SPSS ubican en la cuadrícula del tipo de estilo 26.0 (Statistical Package for Social Scide aprendizaje. La intersección de estos ences). El Excel se usó para la estadístipuntajes se ubica en uno de los cuatro ca descriptiva mientras que para la escuadrantes del plano cartesiano que de- tadística inferencial se usó la aplicación fine el estilo de aprendizaje del estudi- SPSS, en la prueba de Kruskal-Wallis y ante.

el Coeficiente de Cramer.

El rendimiento académico se obtuvo de los promedios finales de las asignaturas Parasitología, Micología, Microbiología sanitaria y Diagnóstico laboratorial obtenidas por los estudiantes de los ciclos $5,6,7$ y 8 respectivamente. El registro de notas se obtuvo de la Dirección Académica de la escuela respectiva, la cual mide el rendimiento académico en la escala vigesimal de 0 a 20, siendo la nota mínima aprobatoria 11.

\section{RESULTADOS}

En base a la aplicación del instrumento se obtuvieron los siguientes resultados: De acuerdo con el inventario de Kolb la mayor cantidad de estudiantes tienen el estilo de aprendizaje asimilador (36\%), sigue el acomodador con $26 \%$, luego el convergente con $21 \% \mathrm{y}$, finalmente, el divergente con 17\%. Ver Figura 1.

El análisis de los datos se llevó a cabo

Figura 1. Distribución de estudiantes según su Estilo de Aprendizaje (EA).

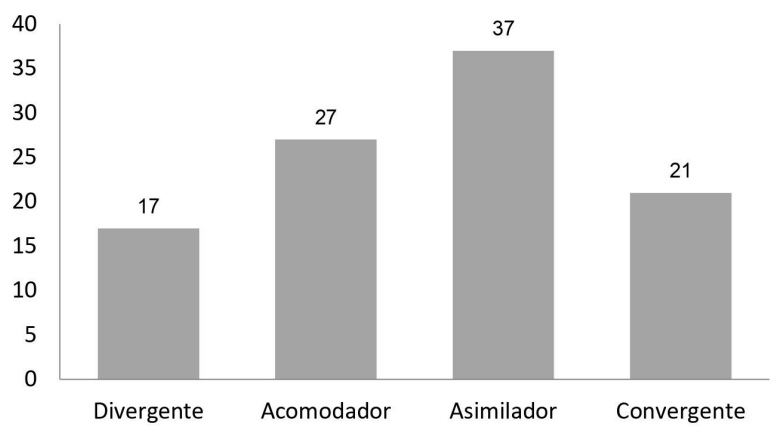

Fuente: Briones, T. (2020) 
En la Figura 2, se muestra que la mayor luego el nivel alto con 25 estudiantes y, cantidad de estudiantes tienen un nivel finalmente, el nivel muy bajo con 23 esde rendimiento académico bajo (28), le tudiantes.

sigue el nivel medio con 26 estudiantes,

Figura 2. Distribución de estudiantes según el nivel de rendimiento académico.

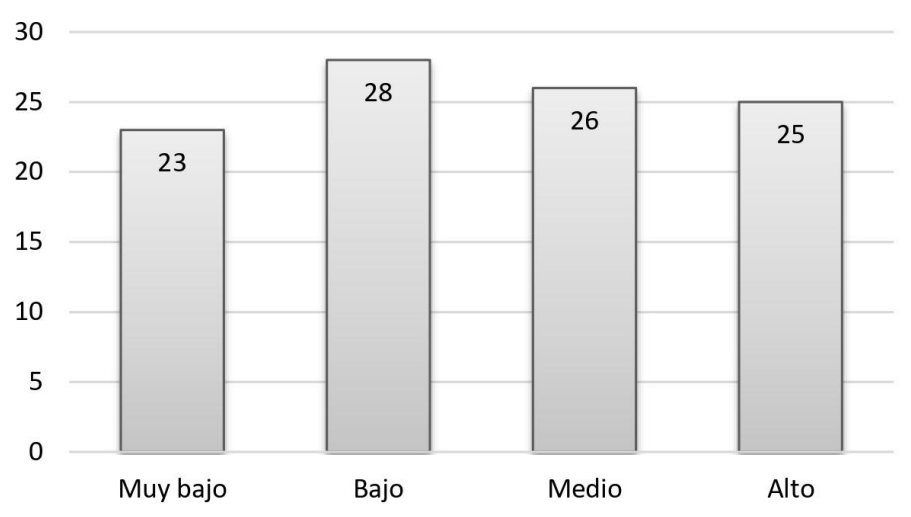

Fuente: Briones, T. (2020)

En la Figura 3, se muestra que la mayor de estudiantes con estilo divergente llecantidad de estudiantes con estilo asim- varon el curso Diagnóstico laboratorial. ilador llevaron el curso de Parasitología, mientras que la cantidad más pequeña 
Influencia del estilo de aprendizaje en el rendimiento académico de estudiantes universitarios

Figura 3. Distribución de estudiantes por curso y estilo de aprendizaje.

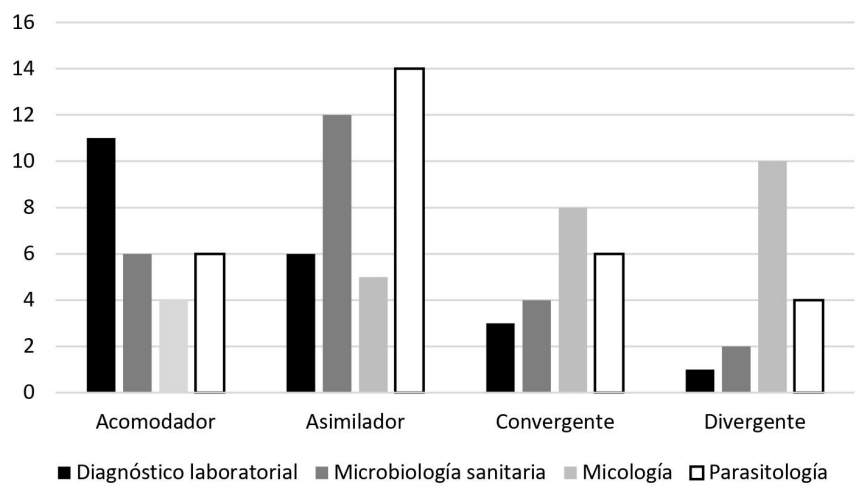

Fuente: Briones, T. (2020)

En la Figura 4, se muestra que en el cur- rendimiento académico alto, y le siguió so de Parasitología estuvieron la mayor el curso de Micología con nueve estudicantidad de estudiantes (10) con nivel de antes del mismo nivel de rendimiento. En la Figura 5, se muestra que la mayor

Figura 4. Distribución de estudiantes por curso y nivel de rendimiento académico.

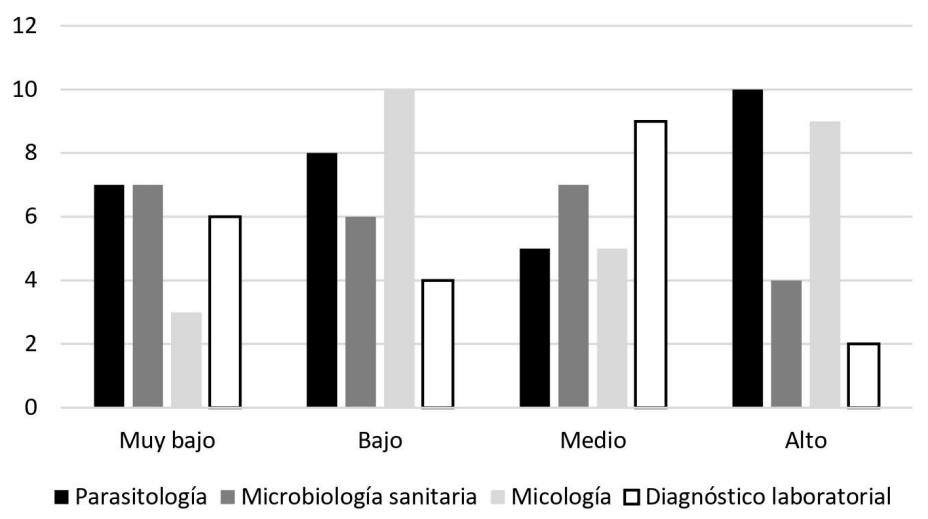

Fuente: Briones, T. (2020)

Revista de Investigación de la Universidad Norbert Wiener, 2020, N. 9 
cantidad de estudiantes con estilo asim- de rendimiento académico alto se enilador (14) tuvieron un nivel de ren- contraron los estudiantes que prefieren dimiento bajo, mientras que en el nivel el estilo convergente (10).

Figura 5. Distribución de estudiantes por estilo de aprendizaje y nivel de rendimiento académico.

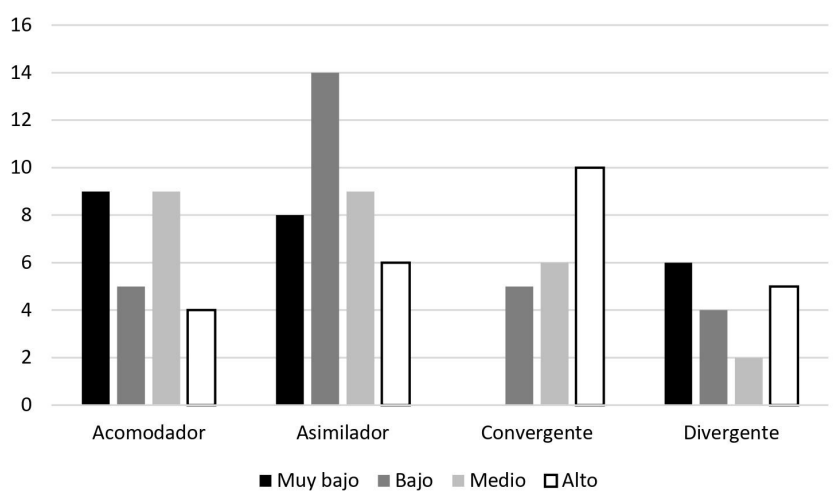

Fuente: Briones, T. (2020)

La Tabla 1 muestra el estadístico de asintótica es 0,007, por lo que se deterprueba $\mathrm{H}=12,264$, la cual ha sido elab- minó que el promedio del curso es diforada con un nivel de significación $\alpha=$ erente en al menos uno de los cuatro es0,05. Asimismo, el valor de significación tilos de aprendizaje del estudiante.

Tabla 1. Estadísticos de prueba ${ }^{\mathrm{a}, \mathrm{b}}$

Promedio del curso

Hde Kruskal-Wallis

gl

Sig. asintótica
12,264

3

0,007

a. Prueba de Kruskal Wallis

b. Variable de agrupación: Estilo de aprendizaje

Fuente: Briones, T. (2020) 
En la Tabla 2 se muestra el estadístico de muestra una relación débil. Cabe menprueba $\mathrm{V}=0,236$ que ha sido trabajado cionar que el estadístico de prueba $\mathrm{V}$ con un nivel de significación $\alpha=0,05$. también fue aplicado para cada uno de También se tiene que la significación los estilos de aprendizaje, y se obtuvo aproximada es de 0,049 , por lo que se los siguientes resultados: acomodador puede determinar que el estilo de apren- $(\mathrm{V}=0,472)$, asimilador $(\mathrm{V}=0,381)$, dizaje está relacionado con el nivel de convergente $(\mathrm{V}=0,463)$ y divergente $(\mathrm{V}$ rendimiento académico; sin embargo, se $=0,212$ ).

Tabla 2. Prueba de relación para estilos de aprendizaje y niveles del rendimiento académico.

\begin{tabular}{cccc}
\hline & & Valor & $\begin{array}{c}\text { Significación } \\
\text { aproximada }\end{array}$ \\
\hline Nominal por & Phi & 0,408 & 0,049 \\
Nominal & V de Cramer & 0,236 & 0,049 \\
N de casos válidos & & 102 & \\
\hline
\end{tabular}

Fuente: Briones, T. (2020)

\section{Discusión}

La respuesta a la hipótesis planteada es que sí existió relación entre los estilos de aprendizaje y el rendimiento académico de los estudiantes de los cursos del área de Microbiología de la especialidad de Laboratorio Clínico y Anatomía Patológica de la Universidad Alas Peruanas del semestre 2019-1. Esto coincide con Serra-Olivares (2017) quien analizó la relación entre estilos de aprendiza- je y rendimiento académico en función de un curso, utilizó el cuestionario CHAEA-36, y a pesar de no encontrarse relación significativa entre estilos de aprendizaje y el curso, al relacionar el rendimiento académico con estilos de aprendizaje demostró que sí existió relación dependiente del estilo de aprendizaje predominante. Juárez et al. (2016) encontraron diferencias significativas con relación a un mayor rendimiento académico por parte de los alumnos 106 Revista de Investigación de la Universidad Norbert Wiener, 2020, N. 9 
cuya preferencia es mayor por los estilos de aprendizaje reflexivo y teórico y por utilizar con una mayor frecuencia estrategias de aprendizaje esta investigación se basó en los cuestionarios CHAEA y CEVEAPEU, es evidente que existe una preferencia por determinado estilo en cada estudiante y esto favorece su desempeño. Quispe-López (2017) encontró que tc es mayor que $\mathrm{t}$ teórica $(12,285>1,96)$, concluyendo que existió una correlación positiva fuerte entre los estilos de aprendizaje y el rendimiento académico de los estudiantes de ingeniería eléctrica de la región Junín. Sin embargo, Colonio-García (2017), al utilizar la prueba de correlación de Rho de Spearman, encuentra que no existe relación entre los Estilos de Aprendizaje y el Rendimiento Académico, de los estudiantes del área de construcción de la Facultad de Ingeniería Civil de la Universidad Nacional de Ingeniería, 2017. Teniendo en cuenta cada estilo de aprendizaje se puede afirmar que sí existe relación significativa entre el estilo de aprendizaje acomodador y los niveles del rendimiento académico y se concluye que al lograr una significación aproximada de 0,035 con $\mathrm{V}$ de Cramer 0,472 existe una relación media de este estilo con el rendimiento académico. Quispe-López (2017) también reportó que si existe una correlación positiva 107 débil entre el estilo de aprendizaje acomodador y el rendimiento académico, en los cursos de formación general y especializada en el semestre académico 2016-II de los estudiantes de Ingeniería Eléctrica de la región de Junín.

Así mismo, se demostró que existe relación significativa entre el estilo de aprendizaje asimilador y los niveles del rendimiento académico al encontrarse que la significación aproximada es de 0,043 con V de Cramer de 0,381, lo que demuestra que existe una relación media de este estilo con el rendimiento académico. Para Quispe-López (2017) también existe una correlación, pero en su caso esta correlación es positiva fuerte, entre el estilo de aprendizaje asimilador y el rendimiento académico, en los cursos de formación general y especializada, en los semestres académicos 2016-II, de los estudiantes de Ingeniería Eléctrica de la región de Junín. La presente investigación también demostró, de acuerdo con el inventario de Kolb, que la mayor cantidad de estudiantes tienen el estilo de aprendizaje asimilador (36\%). Al trabajar con el estilo de aprendizaje convergente se encuentra que la significación aproximada es de 0,041 con $\mathrm{V}$ de Cramer de 0,463, y se demostró que existe relación media entre el estilo de aprendizaje convergente y los niveles del rendimiento académico. Quispe- 
López (2017) también encuentra una correlación positiva media o moderada entre el estilo de aprendizaje convergente y el rendimiento académico, en los cursos de formación general y especializada, en el semestre académico 2016-II de los estudiantes de ingeniería eléctrica de la región de Junín.

Por último, se concluyó que existe una relación débil entre el estilo de aprendizaje divergente y los niveles del rendimiento académico ya que tiene una significación aproximada de 0,048 con $\mathrm{V}$ de Cramer de 0,212. Para Quispe-López (2017) existe una relación media o moderada entre el estilo de aprendizaje divergente y el rendimiento académico en los cursos de formación general y especializada, en los semestres académicos 2016-II, de los estudiantes de Ingeniería Eléctrica de la región de Junín. Para Díaz-Camacho, et. al., (2017) el 4,11\% los estudiantes que se caracterizaron con el estilo divergente-acomodador presentan mejores resultados académicos, mientras que los divergentes $y$ acomodadores (30\% de la población) se comportan de manera similar en el rendimiento académico, y alcanzan un nivel medio y alto. El coeficiente de contingencia para estas variables es de 17,92\%, lo que indica que existe una correlación débil entre las variables rendimiento académico y estilo de aprendizaje según el modelo de Kolb. Lizano, et al. (2015). $\mathrm{Al}$ investigar en estudiantes del quinto año de la carrera de licenciatura en Farmacia de la Universidad de Costa Rica, durante los años 2011 a 2013, aplicó el Cuestionario de Honey-Alonso de Estilos de Aprendizaje y concluyó que los estudiantes no presentan un estilo de aprendizaje puro, sino una combinación de dos o más de estos, lo cual favorece el proceso de aprendizaje al tener más herramientas de adaptación a los requerimientos de los profesores, los cursos y la carrera.

\section{Conclusiones}

En base a la metodología aplicada se demostró que sí existió relación entre los estilos de aprendizaje y los niveles del rendimiento académico de los estudiantes de Laboratorio Clínico y Anatomía Patológica de la Universidad Alas Peruanas del semestre 2019-1.

- Se demostró que existió una relación media entre el estilo de aprendizaje acomodador y los niveles del rendimiento académico de los estudiantes de Laboratorio Clínico y Anatomía Patológica de la Universidad Alas Peruanas de los cursos del área de Microbiología, del semestre 2019-1.

- La relación entre el estilo de apren108 
dizaje asimilador y los niveles del rendimiento académico de los estudiantes de Laboratorio Clínico y Anatomía Patológica de la Universidad Alas Peruanas de los cursos del área de Microbiología del semestre 2019-1 fue media.

- La relación entre el estilo de aprendizaje convergente y los niveles del rendimiento académico de los cursos del área de Microbiología fue media en los estudiantes de Laboratorio Clínico y Anatomía Patológica de la Universidad Alas Peruanas del semestre 2019-1.

- Referente al estilo de aprendizaje divergente y los niveles del rendimiento académico de los cursos del área de Microbiología, se probó que existió una relación débil entre los estudiantes de Laboratorio Clínico y Anatomía Patológica de la Universidad Alas Peruanas del semestre 2019-1.

\section{Sugerencias:}

Que cada docente incorpore diferentes estrategias que aseguren que la sesión de aprendizaje se desarrolle considerando los estilos de aprendizaje, para que cada estudiante pueda mejorar su instrucción desde el estilo de su preferencia y como consecuencia logre un buen rendimiento académico.

Deben incluirse los trabajos en equi109 po, aplicar diferentes enfoques, ejecutar planes (estilo acomodador), lectura, organización de información, análisis (estilo asimilador), desarrollo de casos, solución de problemas, experimentar con nuevas ideas y tomar de decisiones (estilo convergente), fortalecer su capacidad imaginativa, sensibilidad ante los valores, recopilación de información (estilo divergente).

\section{REFERENCIAS BIBLIOGRÁFICAS}

1. Cabrera, J. y Fariñas, G. (2019). El estudio de los estilos de aprendizaje desde una perspectiva vigostkiana: una aproximación conceptual. Revista Iberoamericana de Educación. Recuperado de http:/ / www.rieoei.org/ deloslectores/1090Cabrera.pdf.

2. Chanataxi O.; G. A. (2019) Taxidermia de vertebrados como recurso educativo en el proceso de enseñanza-aprendizaje de la asignatura de anatomía comparada, en la Carrera de Ciencias Naturales y del Ambiente, Biología y Química, Facultad de Filosofía, Letras y Ciencias de la Educación, Universidad Central del Ecuador, 2018-2019. Tesis de Licenciatura. Universidad Central Del Ecuador. http:/ / www.dspace.uce.edu.ec/ 
Influencia del estilo de aprendizaje en el rendimiento académico de es-

tudiantes universitarios

handle/25000/17567

3. Colonio-García, L. A. (2017). Estilos de aprendizaje y rendimiento académico de los estudiantes de los cursos comprendidos dentro de la línea de construcción-DAC-FIC-UNI.

4. Díaz-Camacho, L. M. D., Barrera, H. S., y Castillo, D. J. D. P. R. (2017). Relación entre el Rendimiento Académico en Matemáticas y los Estilos de Aprendizaje de los Estudiantes de la Fundación Universidad Autónoma de Colombia-Fuac. Revista de Estilos de Aprendizaje, 10(20). http://revistaestilosdeaprendizaje.com/article/view/1056

5. Francis, A. \& Segun, M. (2008). Student, teacher and school environment factor as determinations of achievement in senior secondary School Chemistry in Oyo State Nigeria. The Journal of International Social Research, 1(2). Pp.13-34.

6. Gómez, M. (2009). Introducción a la metodología de la investigación científica (2a. ed.), Editorial Brujas. ProQuest Ebook Central, http://ebookcentral.proquest. com/lib/upnortesp/detail.action?docID=3185747.

7. İlçin, N., Tomruk, M., Yeşilyaprak, S. S., Karadibak, D., y Savc1, S. (2018). The relationship between learning styles and academic performance in TURKISH physiotherapy students. BMC medical education, 18(1), 291. https:/ / bmcmededuc.biomedcentral.com/articles/10.1186/s12909-018-1400-2

8. Juárez, C., Rodríguez, G., Escoto, M. \& Luna, E. (2016). Relación de los estilos y estrategias de aprendizaje con el rendimiento académico en estudiantes universitarios. Revista de estilos de aprendizaje, 9(17).

9. Kolb, D. (1984). Experiential Learning. New Jersey. Prentice Hall Inc.

10. Kolb, D. (1999). Inventario de Estilos de Aprendizaje (IEA), Versión 3. Boston. Hay/McBer.

11. Kolb, D. y Kolb, A. (2013). The Kolb Learning Style Inventory - 4.0. A comprehensive guide to the theory, psychometrics, research on validity and educational applications. Recuperado a partir de: http://refhub.elsevier.com/ S2590-2911(20)30006-1/sref29.

12. Lizano, C., Arias, F., Cordero, E. \& Ortiz, A. (2015). Relación entre estilo de aprendizaje y rendimiento académico en estudiantes de far- 
macia de la Universidad de Costa Rica. RIDU, 9(2), 49-63.

13. Quispe-López, C. (2017). Estilos de aprendizaje y rendimiento académico en estudiantes de Ingeniería Eléctrica de la región Junín. (tesis doctoral). Universidad Nacional del Centro del Perú, Junín, Perú. http://repositorio.uncp.edu.pe/handle/ $\mathrm{UNCP} / 4163$.

14. Sarmiento, M. (2007). La enseñanza de las matemáticas y las ntic. Una estrategia de formación permanente. Universitat Rovira I Virgili. ISBN: 978-84-690-8294-2 / D.L: T.16252007

15.Serra-Olivares, J., Valverde, C., Armero, C., \& Madrona, P. (2017). Estilos de aprendizaje y rendimiento académico de universitarios de Educación Física chilenos. RETOS. Nuevas Tendencias en Educación Física, Deporte y Recreación, (32), 62-67. Recuperado a partir de: https://www.redalyc. org/pdf/3457/345751100013.pdf.

16.Tünnermann, C. (2011). El constructivismo y el aprendizaje de los estudiantes. Universidades, (48),2132. [fecha de Consulta 23 de Diciembre de 2020]. ISSN: 00418935. Disponible en: https://

$$
\text { oa?id=373/37319199005 }
$$

17.Vigotsky, L. (1978). Mind in society: the development of higher psychological process. Cambridge, MA: Harvard University Press. 
Influencia del estilo de aprendizaje en el rendimiento académico de estudiantes universitarios

Revista de Investigación de la Universidad Norbert Wiener, 2020, N. ${ }^{\circ}$ 